\section{Revista de CIENCIAS AMBIENTALES Tropical Journal of Environmental Sciences}

Revista de Ciencias Ambientales (Trop J Environ Sci). EISSN: 2215-3896.

(Enero-Junio, 2019) . Vol 53(1): 143-152.

D0l: http://dx.doi.org/10.15359/rca.53-1.8

Open Access: www.revistas.una.ac.cr/ambientales EMAIL: revista.ambientales@una.ac.c Ossenbach Sauter $C$..

\title{
BIOGRAFÍA \\ Charles H. Lankester: \\ Estudioso y conservacionista de orquídeas
}

\author{
Charles H. Lankester: Studious and Conservationist of Orchids \\ Carlos Ossenbach Sauter ${ }^{1}$
}

Palabras clave: Herbario; jardín botánico; naturalista; orquideología.

Keywords: Botanical garden; herbarium; naturalist; orchidology.

\section{Introducción}

Tres grandes nombres forman el más ilustre trío de recolectores en la historia de la orquideología de Costa Rica: Auguste R. Endrés (1838-1874), Alberto Manuel Brenes (1870-1948) y Charles Herbert Lankester (1879-1969).

El primero de ellos fue, sin duda, el más importante botánico en visitar Costa Rica durante el siglo XIX, y quien preparó el primer tratado monográfico (inédito) sobre una familia de plantas costarricenses. Su colección de orquídeas constaba de más de 3500 especímenes, entre los cuales descubrió, describió e ilustró más de 110 especies de orquídeas costarricenses nuevas para la ciencia, las que hoy forman parte importante del herbario del Museo de Historia Natural de Viena. Por fortuna, dicha colección pudo ser recientemente repatriada en forma digital en un esfuerzo conjunto entre el Jardín Botánico Lankester y la prestigiosa institución austríaca.

En las primeras décadas del siglo XX, Brenes, nacido en San

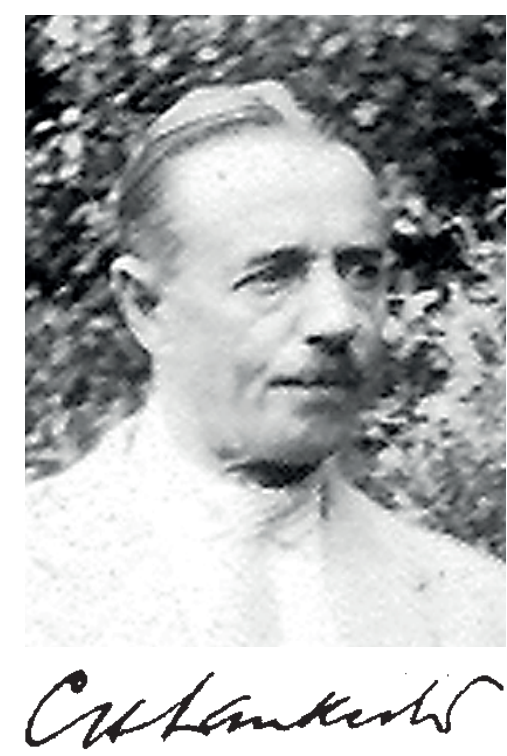
Ramón casualmente en la época en la que Endrés residía en aquella pequeña población al noroeste del Valle Central, realizó extraordinarias colecciones de orquídeas en las montañas circundantes. Entre ellas, el orquideólogo alemán Rudolf Schlechter (1872-1925), con quien Brenes entabló una fructífera relación por intermediación de doña Amparo López-Calleja de Zeledón, la "Gran Dama de la Orquideología Costarricense", describió docenas de nuevas especies en sus Additamenta ad Orchideologiam Costaricensem (1923) en un capítulo titulado Orchidaceae

1 Investigador asociado, Jardín Botánico Lankester, Universidad de Costa Rica, Costa Rica, caossenb@racsa.co.cr

(c)

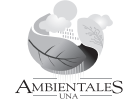




\section{Revista de CIENCIAS AMBIENTALES Tropical Journal of Environmental Sciences}

Revista de Ciencias Ambientales (Trop J Environ Sci). EISSN: 2215-3896.

(Enero-Junio, 2019) . Vol 53(1): 143-152.

DOl: http://dx.doi.org/10.15359/rca.53-1.8

Open Access: www.revistas.una.ac.cr/ambientales

EMAIL: revista.ambientales@una.ac.c Ossenbach Sauter C..

Brenesianae. El herbario de Schlechter en Berlín fue destruido durante un bombardeo en marzo de 1943, pero afortunadamente una parte de los especímenes de Brenes se conservan como duplicados en el herbario de nuestro Museo Nacional.

Por último, Charles Herbert Lankester, especialmente tras la muerte de Schlechter en 1925 y la aparición en escena del gran orquideólogo estadounidense Oakes Ames (1874-1950), se convirtió en la figura dominante de la orquideología costarricense durante el resto de su vida, por lo que heredó con justicia el sobrenombre de "recolector de ojos de lince" con el que el botánico alemán Reichenbach había bautizado a Endrés 50 años antes.

\section{Un inglés en el trópico}

Mejor conocido como "don Carlos", Lankester nació en Southampton, Inglaterra, el 14 de junio de 1879. Después de perder a sus dos padres a la edad de tres años, fue criado, junto con su hermana, por dos de sus tías. Al terminar la escuela secundaria empezó a trabajar en un estudio fotográfico y finalmente se fue a Londres, donde se especializó en fotografía a color en el Instituto Politécnico.

Fue en Londres donde leyó un aviso en el Daily Telegraph, en el que se ofrecía un puesto en Costa Rica a algún joven inglés que quisiera trabajar como asistente en la recién fundada "Sarapiquí Coffee Estates Company". Lankester presentó su solicitud y fue contratado. Pocos meses después desembarcó en Puerto Limón, y siguió por ferrocarril hacia San José; llegó justo a tiempo para tomar parte en el "Baile del Nuevo Siglo", ofrecido por el presidente Rafael Yglesias el 31 de diciembre de 1900 en el Teatro Nacional.

Sarapiquí, en la región Caribe de nuestro país, demostró ser demasiado húmeda para la producción comercial de café, y las plantaciones debieron ser abandonadas tres años después de la llegada de Lankester a Costa Rica. Fue aquí, sin embargo, rodeado por la más exuberante vegetación tropical, donde se despertó su interés por plantas, insectos y aves. Como escribiría el Dr. Louis Williams en su obituario de Lankester en 1969, "don Carlos Lankester llegó al sitio correcto en el momento correcto para participar en la exploración botánica de Costa Rica, quizás el más apasionante sitio biológico en nuestro continente".

Lankester tuvo la oportunidad de conocer, cuando este visitó la región Atlántica, al naturalista suizo Henri Pittier, por entonces director del Instituto Físico-Geográfico, con quien inició una amistad que perduró a través de los años. Muchos de los otros actores en el despertar científico por el que atravesaba Costa Rica en aquellos tiempos, entre ellos Anastasio Alfaro, Paul Biolley, Adolphe Tonduz, Charles Wercklé, los hermanos Curt y Alexander Brade y, años después, Otón Jiménez, fueron de gran importancia para Lankester, y se convirtieron en asiduos visitantes de su finca en Cartago, acompañándole a menudo en sus excursiones botánicas.

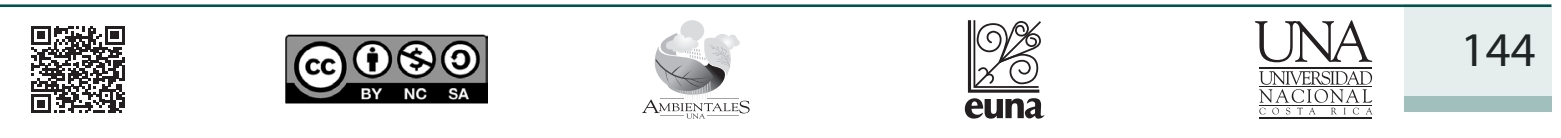




\section{Revista de CIENCIAS AMBIENTALES Tropical Journal of Environmental Sciences}

Revista de Ciencias Ambientales (Trop J Environ Sci). EISSN: 2215-3896. (Enero-Junio, 2019) . Vol 53(1): 143-152. DOl: http://dx.doi.org/10.15359/rca.53-1.8 Open Access: www.revistas.una.ac.cr/ambientales EMAIL: revista.ambientales@una.ac.c Ossenbach Sauter C..

\section{Primeros contactos con nuestras orquídeas: Amor a primera vista}

Cuando su contrato expiró, Lankester regresó a Inglaterra, pero volvió a Costa Rica pocos meses después, llamado por Pittier para hacerse cargo de la Estación Experimental que la United Fruit Company pensaba establecer en Zent, un proyecto que nunca se convirtió en realidad.

Se vio entonces forzado a trabajar con diferentes compañías hasta 1908, cuando regresó a Inglaterra para casarse con Dorothea Mary Hawker, con quien retornó a Costa Rica al aceptar la administración de una finca de café en Cachí, donde vivió durante nueve años con su esposa y joven familia.

Como escribirían Amelia y Philip Calvert unos años más tarde: "la casa de Mr. Lankester estaba bellamente situada a media milla del río Reventazón y alrededor de 150 pies sobre su cauce. No era una casa típica costarricense, ya que - aunque construida en adobe - constaba de dos pisos y no tenía patio. Un gran corredor daba frente a ambas plantas, lugares encantadores, ya que estaban llenos de plantas y en todas partes colgaban canastas de orquídeas, algunas de las cuales estaban en plena floración cuando estuvimos allí” (Calvert y Calvert, 1917) (Figura 1).

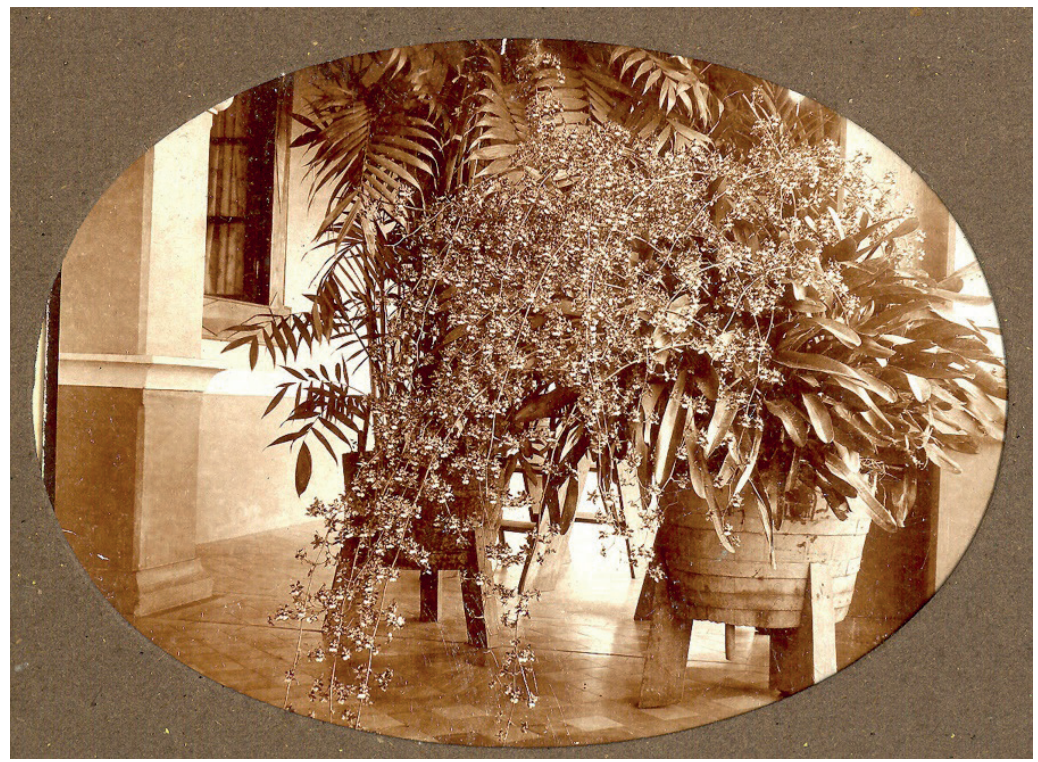

Figura 1. Orquídeas en el corredor de la casa de Lankester en Cachí. Cortesía: Ricardo Lankester

Lankester se interesó inicialmente por aves y mariposas, pero pronto empezaron sus recolecciones de orquídeas en los bosques cercanos, las cuales, en muchas ocasiones, resultaron ser especies nuevas para la ciencia.

Inició correspondencia con Arthur Hill, en ese momento director asistente de los Reales Jardines Botánicos de Kew en 1910, y poco después con Robert Allan Rolfe, la mayor autoridad de Kew en orquídeas. La primera mención suya a orquídeas se encuentra en una carta a Hill de diciembre de 1910, en la que escribió: “Tengo algunas orquídeas, principalmente de interés

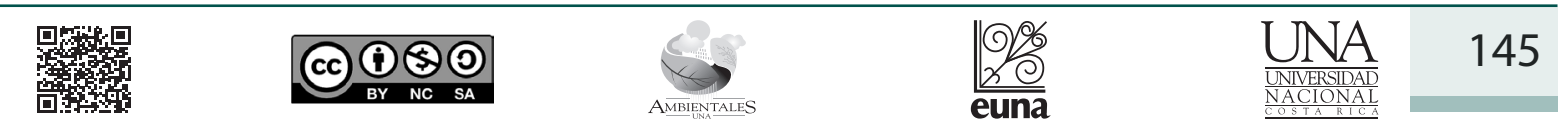




\section{Revista de CIENCIAS AMBIENTALES Tropical Journal of Environmental Sciences}

Revista de Ciencias Ambientales (Trop J Environ Sci). EISSN: 2215-3896.

(Enero-Junio, 2019) . Vol 53(1): 143-152.

Dol: http://dx.doi.org/10.15359/rca.53-1.8

Open Access: www.revistas.una.ac.cr/ambientales EMAIL: revista.ambientales@una.ac.c Ossenbach Sauter C.

botánico, las que le enviaré en cuanto haga más calor del lado suyo". Stelis barbata Rolfe, una planta recolectada cerca de Cachí y enviada a Kew, donde floreció en noviembre de 1912, fue la primera orquídea nueva descubierta por Lankester en Costa Rica. Le seguirían Dichaea ciliolata, Pleurothallis dentipetala y Pleurothallis costaricensis, todas descritas por Rolfe.

Al mismo tiempo, Lankester inició su colección de plantas vivas, que se haría tan famosa con el transcurrir de los años. En 1920 regresó a Inglaterra para matricular a sus cinco hijos, Mary Constance, Helen, Dorothy Anne, John Maurice y Edith, en escuelas inglesas. Su quinta hija, Pamela Joan, nació en Inglaterra ese mismo año. Durante los próximos dos años, realizó investigaciones en plantaciones de café en Uganda, contratado por el gobierno británico.

Cuando regresó a Londres en 1922 se enteró que Rolfe había muerto el año anterior, justo cuando estaba preparando su primer viaje a Costa Rica y a Panamá. Muchas orquídeas que Lankester había enviado a Kew quedaron sin identificación. De este viaje al África regresó a Costa Rica con las primeras semillas de Spathodea campanulata, nuestra "llama del bosque", conocida en inglés, entre muchos otros nombres populares como "Uganda flame". Difícilmente podría suponer él que dicha planta sería clasificada décadas después por la Unión Internacional para la Conservación de la Naturaleza (UICN) dentro de las "100 especies invasoras exóticas más dañinas del mundo".

\section{El nacimiento de un orquideólogo}

Lankester regresó a Costa Rica en 1922, un año crucial para su carrera como orquideólogo, ya que trajo consigo el primer contacto con el profesor Oakes Ames, de la Universidad de Harvard, que representó el inicio de una profunda y larga amistad. Para entender lo que ocurría en el mundo de la orquideología durante aquellos años, hay que recordar que hasta el final de la Primera Guerra Mundial el estudio de las orquídeas era un asunto estrictamente europeo. La orquideología del siglo XIX había sido dominada por un inglés, el gran John Lindley (17901865), seguido por un alemán, Heinrich Gustav Reichenbach hijo (o filius como le gustaba que le llamaran) (1824-1889).

A la muerte de Reichenbach otro inglés, Robert Allen Rolfe (1855-1821) se convirtió en el más destacado orquideólogo mundial, pronto desafiado y reemplazado por otro alemán, esta vez Rudolf Schlechter (1872-1925). No fue sino hasta la muerte de Rolfe en 1921, seguida por la de Schlechter en 1925, cuando el primer experto americano en orquídeas a nivel mundial entraría en escena, en la persona de Oakes Ames (1874-1950), quien ocuparía una posición dominante en el mundo de las orquídeas que nadie pondría en duda, hasta su muerte.

$\mathrm{Al}$ regresar de un viaje a Europa, Ames escribió su primera carta a Lankester, el 17 de setiembre de 1922, en la que expresaba: "En Kew encontré muchos especímenes recolectados por usted en Costa Rica, la mayor parte sin determinar. En vista de que a Kew le tomará un tiempo recuperarse de la muerte de Rolfe y de que los alemanes están haciendo grandes esfuerzos para obtener material costarricense a través de Wercklé, Jiménez y Tonduz, me pareció que usted podría estar dispuesto a cooperar conmigo estimulando el interés por las orquídeas entre sus vecinos." Y

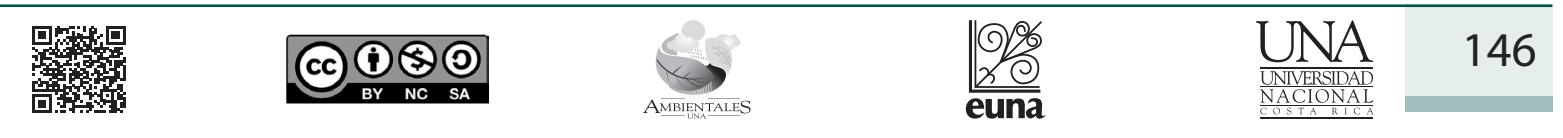




\section{Revista de CIENCIAS AMBIENTALES Tropical Journal of Environmental Sciences}

Revista de Ciencias Ambientales (Trop J Environ Sci). EISSN: 2215-3896.

(Enero-Junio, 2019) . Vol 53(1): 143-152.

D0l: http://dx.doi.org/10.15359/rca.53-1.8

Open Access: www.revistas.una.ac.cr/ambientales EMAIL: revista.ambientales@una.ac.cr

continuaba: "Sería seguramente provechoso si usted pudiera enviarme ejemplares de herbario y enviar recolectores a las regiones ricas en orquídeas en Costa Rica. Ya he identificado y descrito como nueva una de sus especies de Pleurothallis. Me refiero a P. palliolata.

Terminaba Ames esta famosa primera carta a Lankester diciendo: "Tenemos que trabajar rápido si queremos mantenernos delante de los alemanes. Me sorprendió ver los esfuerzos que han hecho para asegurarse un monopolio de las especies tropicales americanas". Recordemos que durante esos años Schlechter estaba describiendo cientos de especies nuevas de América Central provenientes de las recolecciones de Charles Powell en Panamá y de Wercklé, Tonduz, Brenes, Acosta y Jiménez en Costa Rica.

Lankester contestó la carta de Ames inmediatamente y se convirtió pronto en el recolector favorito del gran experto estadounidense. A lo largo de los siguientes quince años, Ames describió más de 100 especies de orquídeas nuevas para la ciencia, entre los especímenes que Lankester le enviaba constantemente desde Costa Rica. En su carta de respuesta a Ames, fechada el 11 de octubre de 1922, Lankester escribía: "Me temo que tratar de estimular el interés por las orquídeas en Costa Rica tendrá poco éxito... creo que solo yo tengo la locura necesaria." Las orquídeas se habían convertido en el centro del mundo de Lankester, y lo seguirían siendo hasta el último día de su vida.

En 1922, Ames inició una serie de publicaciones con el título de "Schedulae Orchidianae". En su tercer fascículo, en enero de 1923, empezó a publicar las descripciones de las orquídeas de Lankester que habían quedado sin determinar en Kew después de la muerte de Rolfe. Le siguieron luego las especies que Lankester empezó a enviar desde Costa Rica, y una de ellas dio lugar a una famosa anécdota. "Su número 387 no es una orquídea, es una bromelia", escribió Ames en 1923. Lankester contestó enseguida: "Véala de nuevo." ¡Un inusual nuevo género de Orchidaceae había sido descubierto! Ames admitió su error y una semana después escribió a Lankester: "Parece haber un nuevo género entre sus especímenes, Lankesterella sería un buen nombre" (Figura 2).

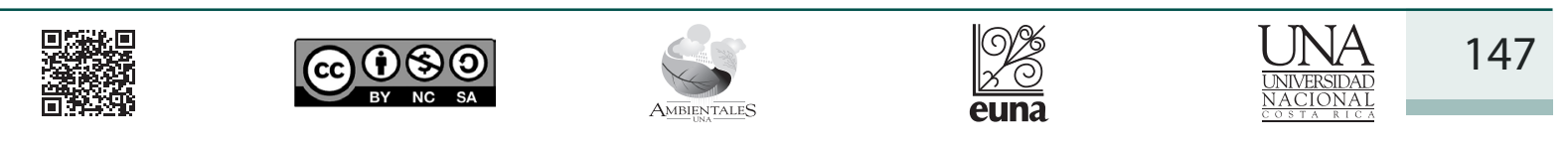




\section{Revista de CIENCIAS AMBIENTALES Tropical Journal of Environmental Sciences}

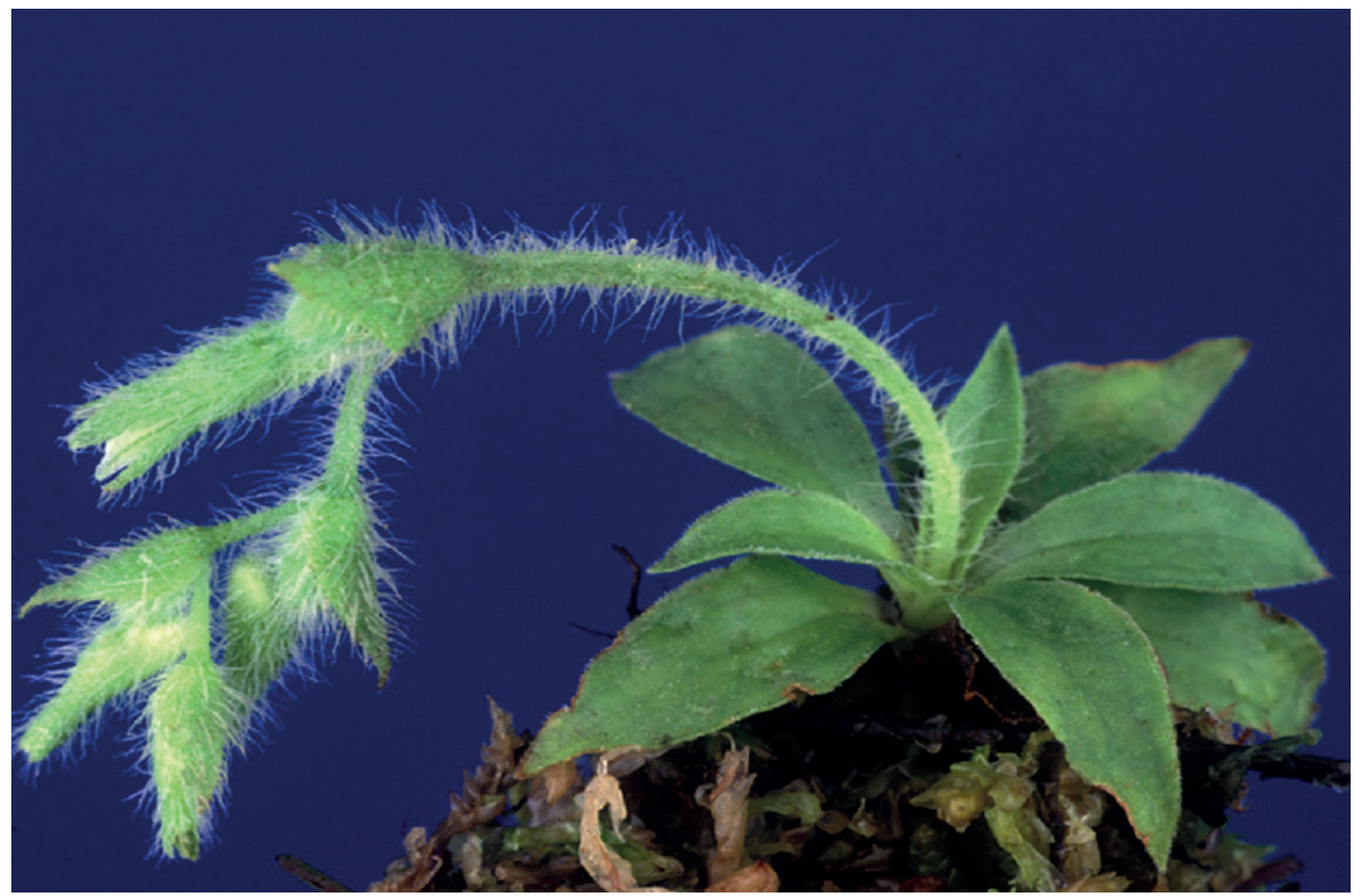

Figura 2. Aspecto de Lankesterella orthantha. Fotografía: Franco Pupulin.

\section{Prosperidad e incertidumbre}

Después de la Primera Guerra Mundial, los precios para las materias primas tropicales, tales como el café y el banano, subieron rápidamente. Las restricciones impuestas por cuatro años de guerra naval fueron ahora reemplazadas por una demanda creciente. Los sectores cafetaleros costarricenses tuvieron uno de sus más prósperos períodos en la década de los 1920, y Lankester no fue el único en aprovechar la situación. En 1924 se trasladó a "Las Cóncavas", una finca cafetalera que había adquirido cerca de Cartago.

El café había sido el motivo de su llegada inicial a Costa Rica en 1900, y dependería del café durante el resto de su vida. Su colección de orquídeas ya era enorme. En agosto de 1924 escribía a Ames: “... Esperamos mudarnos a la finca a mediados de noviembre; esto significa, entre otras cosas, trasladar cerca de 1800 orquídeas por caminos inimaginables." Se entiende, entonces, porqué la finca de Lankester se convertiría, con el paso de los años, en la Meca de botánicos y orquideólogos de todo el mundo.

Con su excepcional colección de plantas, "Las Cóncavas" no podía pasar inadvertida para naturalistas del mundo. Los jardines contaban con importantes colecciones de cactus, palmeras

\begin{tabular}{|c|c|c|}
\hline 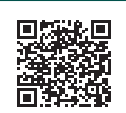 & (c) (i) (3) (2) & 148 \\
\hline
\end{tabular}




\section{Revista de CIENCIAS AMBIENTALES Tropical Journal of Environmental Sciences}

Revista de Ciencias Ambientales (Trop J Environ Sci). EISSN: 2215-3896.

(Enero-Junio, 2019) . Vol 53(1): 143-152.

D0l: http://dx.doi.org/10.15359/rca.53-1.8

Open Access: www.revistas.una.ac.cr/ambientales EMAlL: revista.ambientales@una.ac.cr Ossenbach Sauter C..

y bromelias, aunque las orquídeas fueron siempre las favoritas de Lankester. Entre los visitantes de "Las Cóncavas" estuvieron Arthur Hill -director de los Jardines Botánicos de Kew-, Thomas Barbour -de la Universidad de Harvard-, Harvey Stork, William Maxon, Wilson Popenoe, Philip Calvert, James Rehn, Paul C. Standley y Louis O. Williams.

El año 1925 nos muestra a Lankester en lo que parecía ser la cima de su carrera como cafetalero y orquideólogo. Visitó a sus hijos durante un corto viaje a Inglaterra, su finca estaba en plena producción, y en julio de ese año fue electo miembro honorario de la American Orchid Society. ¿Cuál era, sin embargo, la verdadera situación de Lankester? Parece que había problemas de los que nunca se habló.

Ya en 1924 había intentado convencer a Ames para que invirtiera en su finca, una propuesta que Ames cortésmente declinó. Luego, en noviembre de 1925, parecía decidido a vender todo y abandonar Costa Rica: "Es muy posible que la próxima semana culmine la venta de este lugar, y si así fuese, Costa Rica no me verá nunca más." Es probable que nunca sepamos qué sucedió, pero afortunadamente Lankester no vendió y no abandonó el país, aunque aparentemente no dejó de tener problemas económicos hasta el fin de sus días. Continuó enviando orquídeas a Ames, ahora adjuntando a menudo crudos bosquejos o fotografías, que se conservan hoy en el Herbario de Orquídeas de Oakes Ames, en la Universidad de Harvard.

Un visitante muy importante llegó a Costa Rica en enero de 1924, y de nuevo en diciembre de 1925, Paul Carpenter Standley (1884-1963). Tres años antes, Standley, botánico del Museo Nacional de los Estados Unidos, había arribado al puerto de La Libertad, El Salvador, la primera visita a Centroamérica de quien se convertiría probablemente en el personaje más importante en la exploración botánica de la región durante la primera mitad del siglo XX.

Durante más de 40 años Standley recolectó en forma intensiva en todos los países de América Central y publicó una serie de obras fundamentales sobre sus floras. Adquirió un conocimiento íntimo de la región, no solamente en sus aspectos botánicos, sino también sobre su cultura y tradiciones. Fue amigo de toda la comunidad científica centroamericana de la época y contribuyó como nadie al desarrollo del estudio y la investigación de la botánica, al crecimiento de los herbarios existentes y a la creación de muchos otros nuevos. Standley hizo gran amistad con Lankester, quien le visitó en algunas ocasiones en la Escuela Agrícola Panamericana "El Zamorano", en Honduras, en cuyo desarrollo Standley jugó un papel importante. Mantuvieron una estrecha amistad hasta la muerte de Standley en 1963.

Entre 1923 y 1925 Standley realizó dos visitas a Panamá, que fueron seguidas por extensas giras de recolección por nuestro país, realizadas en los primeros meses de 1924 y nuevamente entre diciembre de 1925 y marzo de 1926. Recolectó una enorme cantidad de material costarricense -más de 15,000 especímenes- entre ellos más de 30 orquídeas nuevas para la ciencia.

En noviembre de 1923 Ames ponía a Lankester sobre aviso: "Standley está en Nueva York el día de hoy. Cuando llegue a Costa Rica haga que lluevan orquídeas sobre él. Asegúrese que ninguna orquídea escape de la prensa." En el prólogo a su "Flora de Costa Rica" Standley recordaba el gesto de Lankester en diciembre de 1925, mientras se encontraba en gira por Santa María de Dota: " $E l$

\begin{tabular}{|c|c|c|c|c|c|}
\hline 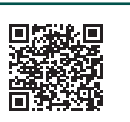 & (c) (i) (3) (2) & $\Leftrightarrow$ & $\frac{O O F}{2 \%}$ & 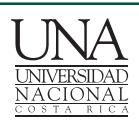 & 149 \\
\hline
\end{tabular}




\section{Revista de CIENCIAS AMBIENTALES Tropical Journal of Environmental Sciences}

Revista de Ciencias Ambientales (Trop J Environ Sci). ElSSN: 2215-3896.

(Enero-Junio, 2019) . Vol 53(1): 143-152.

DOl: http://dx.doi.org/10.15359/rca.53-1.8

Open Access: www.revistas.una.ac.cr/ambientales

EMAIL: revista.ambientales@una.ac.c Ossenbach Sauter C..

autor nunca olvidará el gesto [de los Lankester], que enviaron un mensajero especial en un viaje de dos días para traerme un presente en tiempo de Navidad".

A finales de 1932, ya de regreso en Costa Rica, Lankester fue nombrado en la Junta Directiva del Museo Nacional por el Presidente de la República, un gran honor que compartió con otras famosas figuras en la historia de las orquídeas en Costa Rica, como Anastasio Alfaro y doña Amparo de Zeledón. De nuevo en el extranjero, visitó las islas Canarias en 1934, explorando Tenerife con el conocido jardinero y profesor Juan Bolinaga, director del Jardín Botánico de la isla. El año siguiente viajó a Brasil, y después de atravesar el continente salió hacia el Pacífico por Bolivia.

Por esa época Ames describió las últimas tres orquídeas recolectadas por Lankester en Costa Rica. Fueron publicadas en los "Harvard University Botanical Museum Leaflets" y se trató de tres diferentes especies de Stelis: S. crystallina, S. latipetala y S. transversalis. Al final de una década marcada por una enorme indecisión por su futuro, dividido entre el amor por su país de adopción -a pesar de las dificultades- y con nostalgia tanto por su patria como por sus hijos, Lankester volvió a Inglaterra antes de que iniciara la Segunda Guerra Mundial.

Regresó a Costa Rica al final de 1939, escapando milagrosamente de un torpedo alemán en el convoy en el que cruzaban el Atlántico; un pequeño barco al lado del suyo fue la infortunada víctima. De acuerdo con nuestros registros, Lankester envió el último paquete de plantas a Ames en marzo de 1942, aunque su correspondencia continuó hasta la muerte de Ames en 1950. Curiosamente, nunca llegaron a conocerse en persona.

\section{Su espíritu conservacionista}

El interés de Lankester por la conservación de la naturaleza fue siempre manifiesto, y quizás más evidente en esta etapa de su vida. De especial interés fue para él el inicio -por motivos de estrategia militar relacionados con el temor a una invasión japonesa en nuestra costa del Pacífico- de la carretera Panamericana en su sector sur, que tendría un enorme impacto ambiental en grandes zonas de la Cordillera de Talamanca.

Se interesó tanto por este asunto, que en 1943 fue nombrado por Mariano Montealegre Carazo, Secretario de Agricultura, para formar parte de una comisión especial de notables que debería valorar aspectos relacionados con la conservación y estudio de los excepcionales bosques de robles (Quercus spp.), mayoritariamente Quercus copeyensis, existentes en el trayecto en construcción. Los otros miembros de dicha comisión fueron Anastasio Alfaro González, Clodomiro Picado Twight, Jorge León Arguedas y Rafael Chavarría Flores.

Por otra parte, el famoso ornitólogo y conservacionista estadounidense Alexander F. Skutch (1904-2004), quien vivió en Costa Rica desde 1935 hasta su muerte, contrajo matrimonio en 1950 con Pamela, hija de Lankester. Skutch había recolectado orquídeas en Guatemala en 1933 y 1934, por lo cual no es de extrañar que al llegar a Costa Rica pronto conociera a Lankester. Skutch y su esposa vivieron el resto de sus días en una pequeña finca a orillas del río Peñas

\begin{tabular}{|c|c|c|}
\hline 句8 & (c) (i) (3) (2) & 150 \\
\hline
\end{tabular}




\section{Revista de CIENCIAS AMBIENTALES Tropical Journal of Environmental Sciences}

Revista de Ciencias Ambientales (Trop J Environ Sci). ElSSN: 2215-3896.

(Enero-Junio, 2019) . Vol 53(1): 143-152.

Dol: http://dx.doi.org/10.15359/rca.53-1.8

Open Access: www.revistas.una.ac.cr/ambientales

EMAIL: revista.ambientales@una.ac.c Ossenbach Sauter C..

Blancas que Skutch llamó "Los Cusingos", por el nombre local del tucancillo pico naranja (Pteroglossus frantzii). En verdad, las relaciones entre Skutch y su familia política nunca fueron las mejores, ya que los Lankester reclamaron siempre a Skutch las condiciones de aislamiento y extrema frugalidad en las que Pamela vivió el resto de sus días.

A inicios de la década de 1940, Lankester empezó su último gran proyecto: un libro que sería su tributo a las orquídeas de su país adoptivo y que llevaría el título de "Costa Rican Orchids". Pero Lankester no solo quería escribir un libro. También soñaba con reunir fondos para convertir su finca en un paraíso para la conservación de las orquídeas costarricenses. Trató de interesar a la American Orchid Society en el proyecto, pero no encontró apoyo suficiente. Los años transcurrieron y el libro nunca se publicó en vida de don Carlos. No fue sino hasta en 2013 que el Jardín Botánico Lankester, de la Universidad de Costa Rica, lo publicó en forma póstuma.

En 1949 Lankester tomó la irreversible decisión de permanecer en Costa Rica por el resto de su vida. Como escribía a Gordon Dillon, de la American Orchid Society en 1960: "Ni idea de regresar a Inglaterra, vendí mi casa en Londres en 1949, pero mantengo un lazo estrecho a través de tres de mis hijas casadas allí. Un verano como el último ciertamente es invitador, pero los inviernos...".

En 1955, después de la muerte de su esposa y a la edad de 76 años, Lankester decidió vender su finca, pero conservó su jardín, una parte del terreno de 11 hectáreas llamada "El Silvestre". Se trasladó a vivir a la capital, a una casa que había comprado en Moravia. Unos años más tarde, en junio de 1961, en una ceremonia en la embajada británica en San José, le fue conferida la Orden del Imperio Británico por orden de la Reina Isabel II, un honor del cual Lankester estaría orgulloso hasta el fin de sus días.

Cabe destacar que "El Silvestre" fue heredado por su hija Dorothy (1912-1992), pero las dificultades económicas hacían imposible conservar el lugar como el paraíso de orquídeas que Lankester deseaba. Ella se vio forzada a vender la propiedad. Sin embargo, gracias a los esfuerzos de la Asociación Costarricense de Orquideología, del orquideólogo Rafael Lucas Rodríguez y un grupo de socios de la American Orchid Society, más el apoyo del Stanley Smith Horticultural Trust de Inglaterra, se logró reunir el dinero necesario. La propiedad fue comprada y donada a la Universidad de Costa Rica. El 2 de marzo de 1973 se inauguró oficialmente el Jardín Botánico Lankester.

El sueño de don Carlos se había hecho al fin realidad. Hoy ese santuario de orquídeas se ha convertido en el más importante centro de investigación de la familia Orchidaceae en el continente americano. Los investigadores del Jardín Lankester han descubierto desde su fundación más de 300 especies de orquídeas costarricenses - nuevas para la ciencia- y el antiguo "El Silvestre" de don Carlos es nuevamente "la Meca" de profesionales de la ciencia y de la orquideología de todo el mundo.

Lankester falleció el 8 de julio de 1969, en compañía de su hija Dorothy, tras soportar problemas económicos hasta los últimos días.

Tal fue la plenitud con que vivió, que en una conversación con el botánico Otón Jiménez, le manifestó: "Estoy muy agradecido con Costa Rica, porque puedo decir que he sido aquí muy feliz.

\begin{tabular}{|c|c|c|c|}
\hline 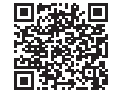 & (c) (i) (3) () () & $\Leftrightarrow$ & 151 \\
\hline
\end{tabular}




\section{Revista de CIENCIAS AMBIENTALES Tropical Journal of Environmental Sciences}

Revista de Ciencias Ambientales (Trop J Environ Sci). EISSN: 2215-3896.

(Enero-Junio, 2019) . Vol 53(1): 143-152.

DOl: http://dx.doi.org/10.15359/rca.53-1.8

Open Access: www.revistas.una.ac.cr/ambientales

EMAlL: revista.ambientales@una.ac.cr Ossenbach Sauter C.

No ciertamente porque lograra cuantiosos bienes materiales, pues mi fortuna es modesta. Tampoco me he escapado de afrontar situaciones difíciles de salud, ni de familia, ni de reveses de negocios, ni las mil calamidades propias de nuestro paso por la vida, pues de todo he tenido, incluso el dolor de ver partir a la Gloria Eterna a mi abnegada esposa, tan delicada y cariñosa. Pero debo agradecer al buen Dios que me ha dado larga vida para disfrutar plenamente de esta maravillosa naturaleza de Costa Rica. ¿Qué mayor felicidad podría yo esperar?».

El gran botánico Louis O. Williams, gran amigo de Lankester durante largos años, escribió en su obituario: "Generoso hasta lo último, hospitalario para todos, fue consejero para todos los cientificos que llegaron a Costa Rica. Sus intereses fueron universales: mariposas, aves, pero especialmente plantas epífitas- orquídeas, bromelias y aroides". Y el ya citado Standley agregó: "Un naturalista en el mejor y más amplio sentido de la palabra".

\section{Referencias}

Calvert, A. S. \& Calvert, P. P. (1917). A Year of Costa Rican Natural History. New York: MacMillan Company.

Jiménez, O. (Octubre, 1967). Charles Herbert Lankester. Revista de Agricultura, 248-264.

Ossenbach, C. (2013). Charles Herbert Lankester (1879-1969): His life and legacy. Lankesteriana, 13(3), 359-374.

Ossenbach, C. (2016). Orquídeas y orquideología en América Central: 500 años de historia. Cartago: Editorial Tecnológica de Costa Rica.

Standley, P. C. (1925). Orchid collecting in Central America. Smithsonian Report for, 1922, 309328. Washington.

Urbina V. S. (2005). Un científico costarricense de origen inglés: Charles Herbert Lankester (1879-1969). Revista Biocenosis, 19(2), 9-11.

Williams, L. O. (1969). In Memoriam: Charles Herbert Lankester - 1879-1969. (AOS) Bulletin, $38,860-862$.

(cc)

